ges n'ayant pas débarrassé la caséine de l'alcool qui l'avait imprégnée, parce qu'il y était resté sous la forme d'éther éthylique.

Dans nos dernières préparations, nous substituions à l'éther, l'acétone. C'est un corps neutre qui ne peut pas donner d'éther avec la caséine, qui bout à basse température et qui peut même tout à la fois se substituer à l'alcool et à l'éther, si bien qu'il n'y a qu'un seul solvant à employer.

Dans la préparation de la caséine, un des avantages de l'éther, c'est d'être un solvant antiseptique et la caséine pulvérulente que l'on obtient à la fin des opérations est stérile, et par conséquent, peut être employée, en observant toutes les précautions que les recherches microbiologiques imposent, pour faire des solutions qui n'auraient pas besoin d'être stérilisées.

L L'acétone jouit de propriétés a nalogues, ce qui est une raison de plus à ajouter aux précédentes pour justifier son emploi dans la préparation de la caséine au laboratoire.

Conclusions. - I. - L'acétone est un solvant qui mérite d'être utilisé dans l'analyse biologique du lait.

II. - Une méthode qui viserait à doser volumétriquement la caséine dans le lait par précipitation de celle-ci avec l'acétone seul ou l'acétone acide ne nous paraît pas recommandable jusqu'ici.

III. - Alors que l'alcool acétique ne précipite que la caséine dans le lait et laisse dans le sérum l'albumine, l'acétone acétique précipite presque la totalité des protéines du lait.

IV. - L'acétone peut être substitué à l'alcool et à l'éther dans la préparation de la caséine pure au labor.ıtoires.

\title{
TRAITEMENT DU LAIT PAR LA CHALEUR
}

\section{(Stassanisation)}

8 ${ }^{\circ}$ RAPPORT DE LA LAITERIE D'ESSAIS DE L'ETAT,

A HILLEROD (Danemark)

(Fin)

CONSOMMATION D'EAU DE L'APPAREIL A STASSANISER. - La consommation d'eau nécessaire pour refroidir le lait dépend tout d'abord de la température à laquelle on désire refroidir le lait, qui dépend à son tour de l'utilisation à donner au lait ; mais comme il s'agit de chauffer du lait destiné à la eonsommation, il est naturel de refroidir le lait à la température la plus basse possible que permet l'eau de fontaine employée : c'est sur cette base que les mesures de consommation d'eau furent effectuées.

Ici, la température de l'eau de fontaine est de 8 à $9^{\circ} \mathrm{C}$. $\left(46^{\circ} 4\right.$ à 
$48^{\circ} 2 \mathrm{~F}$.). Le tableau I montre, suivant la température de l'eau et du lait, les quantités d'eau employées pour refroidir des quantités de lait déterminées.

Le refroidissement doit être considéré comme satisfaisant, car a vec une quantité d'eau double du lait, on est arrivé à refroidir le lait à $2^{\circ} \mathrm{C}$. au-dessus de la température de l'eau, et il est pratiquement impossible d'obtenir plus, d'autant plus que le refroidissement est très court.

Tableau I.

\begin{tabular}{|c|c|c|c|c|c|c|c|c|}
\hline \multirow{2}{*}{$\begin{array}{l}\text { Essai } \\
\text { No }^{\circ}\end{array}$} & \multicolumn{3}{|c|}{ Température de l'eau } & \multicolumn{3}{|c|}{ Température du lait } & \multirow{2}{*}{$\begin{array}{l}\text { Lait } \\
\text { Kgs }\end{array}$} & \multirow{2}{*}{$\begin{array}{l}\text { Eau } \\
\mathrm{Kgs}\end{array}$} \\
\hline & Entrée & Sortie & $\begin{array}{c}\text { Augmen- } \\
\text { tation }\end{array}$ & Entrées & Sorties & Pertes & & \\
\hline 1 & 8,5 & 22,5 & 14,0 & 27 & $13, \tilde{5}$ & 13,5 & 840 & 840 \\
\hline 2 & 9 & 22,5 & 13,0 & 26 & 13 & 13,0 & 840 & 840 \\
\hline 3 & 8 & 20,0 & 12,0 & 26 & 12,5 & 13,5 & 840 & 1000 \\
\hline 4 & 9 & $2 C, 0$ & 11,0 & 28 & 13 & 15,0 & 840 & 1200 \\
\hline 5 & 9 & 18,0 & 9,0 & 29 & 11 & 18,0 & 840 & 1700 \\
\hline 6 & 8 & 16,0 & 8,0 & 26 & 10 & 16,0 & 840 & 1700 \\
\hline 7 & 9 & 15,5 & 6,5 & 29 & 10,5 & 18,5 & 840 & 2500 \\
\hline 8 & 8 & 13,0 & 5,0 & 26 & 10 & 16,0 & 840 & 2600 \\
\hline
\end{tabular}

Si on désire un refroidissement plus intense, il faut employer de la saumure à basse température, ce qui est facile.

\section{CONSOMIMATION DE FORCE DE L'APPAREIL A STASSANISER ET DURÉE DU TRAITEMENT}

Pendant les essais, l'appareil à stassaniser était fixé de telle façon que l'arbre commandant les pompes de l'a ppareil pouvait être actionné par un moteur électrique muni d'un ampèremètre et d'un compteur, de telle sorte qu'il était facile de noter la consommation de force correspondant aux diverses capacités de l'appareil, et celle-ci a été plutôt régulière. Quand la pompe de pression de lait de l'appareil travaillait à la pression normale de 4-5 atmosphères et que l'appareil avait sa capacité normale de 840 litres (187 gallons) par heure, la consommation de force était de 1,4 à 1,6 chevaux.

La durée du traitement, c'est-à-dire l'es pace de temps compris entre le moment où le lait entre dans l'appareil et celui où il le quitte, a été, à capacité normale, et sous pression de 4 atmosphères, de 2 minutes 5 à 2 minutes 7, réparties comme suit sur les sections individuelles de l'appareil : 
environ 28 mètres (env. 91 pieds) Régénérateur (tuyaux intérieurs) a vant la pompe ............... » 20 (env. 65 pieds) Stassaniseur a près la pompe .......... 1) 28 (env. 91 pieds) Régénérateur (tuyaux extérieurs) a près la pompe.............. ) 28 " (env.91 pieds) Retroidisseur..

environ 104 mètres (env. 338 pieds) Total ...... en secondes<smiles>[Se][Se]</smiles>

14- 17 is arist iangisati

$28-31$

$48-52$

Comme la longueur totale des tuyaux à travers laquelle le lait a à passer est de 104 mètres (env. 338 pieds) (le lait devant passer deux fois dans le régénérateur), la vitesse moyenne est d'environ $0 \mathrm{~m} .67$ par seconde, mais elle diffère considérablement dans les diverses sections a vant et a près la pompe.

Dans les tuyaux intérieurs du régénérateur, on l'a mesurée à environ $0 \mathrm{~m}$. 5 par seconde avant la pompe, et à $1 \mathrm{~m} .25$ par seconde a près la pompe dans le stassaniseur, $0 \mathrm{~m}$. 9 par seconde dans les tuyaux extérieurs du régénérateur et env. 0 m. 5 par seconde dans le refroidisseur.

Ces mesures, qui ont été effectuées aussi exactement qu'il est possible de le faire dans la pratique, aussi bien directement qu'avec des dissolutions d'iode comme indieateur, montrent que, dans l'ensemble, la durée du traitement est très courte, particulièrement courte à la haute température du stassaniseur, et c'est probablement à ce fait, joint à ee que le traitement a lieu en tuyauterie férmée, sans admission d'air, que la composition naturelle du lait doit d'être si peu modifiée par le traitement.

\section{EFFET DE LA STASSANISATION SUR L'ÉTAT PHYSIQUE ET CHIMIQUE DU LAIT}

odeur, saveur et APPARENCE, - Quand le lait stassanisé quitte I'appareil, son a pparence n'est pas différente de celle du lait non traité entrant dans l'appareil. L'odeur et la saveur du lait ne sont pas non plus modifiées. Ceci a été confirmé par une longue suite de dégustations : on a pris par exemple 1 litre (environ 0 gallon 22) du même lait, a vant et a près le traitement, qu'on a placé dans des ballons de verre propres. On a ensuite demandé à diverses personnes, expertes ou non, de décider, par l'odeur et le goût, quel était celui qui avait été chauffé et celui qui ne l'a vait pas été. Ces dégustations ont donné des résultats très différents et très variables, avec des avis négatifs et positifs en proportions à peu près égales, de sorte qu'il n'a pas été possible, par 
ce moyen, de trouver de différence entre le lait chauffé et le lait non chauffé.

monté de LA créme. - Pour les recherches relatives à la montée de la crème, diverses méthodes ont été employées, la température de la erème pendant la montée, ainsi que la forme et la dimension des récipients étant différents. Dans un but de comparaison, du lait brut et du lait pasteurisé ont été expérimentés dans les mêmes conditions.

Les résultats des essais sont ceux indiqués dans le tableau ci-dessous. La température tempérée de la chambre a été de $15^{\circ}-20^{\circ} \mathrm{C}$. (environ $59^{\circ} 0-68^{\circ} 0 \mathrm{~F}$.) et la température de la chambre froide d'environ $6^{\circ} \mathrm{C}$. (4208 F.). La durée du repos du lait pendant la montée de la crème a été de 24 heures.

HIFERES PROPORTIONNELS DE LA MONTÉE DE LA CRĖME DANS DES CONDITIONS DIFFÉRENTES.

\begin{tabular}{|c|c|c|c|c|c|c|c|c|}
\hline \multirow[b]{2}{*}{ GROUPE } & \multicolumn{2}{|c|}{$A=I: I$} & \multicolumn{2}{|c|}{$B=2: I$} & \multicolumn{2}{|c|}{$\mathrm{C}=12: \mathrm{J}$} & \multicolumn{2}{|c|}{$D=50: I$} \\
\hline & $\begin{array}{l}\text { Chambre } \\
\text { tempérée }\end{array}$ & $\begin{array}{c}\text { Chambre } \\
\text { froide }\end{array}$ & $\begin{array}{l}\text { Chambre } \\
\text { tempérée }\end{array}$ & $\begin{array}{c}\text { Chambre } \\
\text { froide }\end{array}$ & $\begin{array}{l}\text { Chambre } \\
\text { tempérée }\end{array}$ & $\begin{array}{c}\text { Chambre } \\
\text { froide }\end{array}$ & $\begin{array}{l}\text { Chambre } \\
\text { tempérée }\end{array}$ & $\begin{array}{c}\text { Chambre } \\
\text { froide }\end{array}$ \\
\hline $\begin{array}{l}\text { it brut ... } \\
\text { it stassa- }\end{array}$ & 9,1 & 8,9 & 9,2 & 10,1 & 9,0 & 11,7 & 6 & 16 \\
\hline $\begin{array}{l}\text { nisé ...... } \\
\text { it pasteu. } \\
\text { isé à haute }\end{array}$ & 6,7 & 7,9 & 6,2 & 6,8 & 4,2 & 4,0 & 4 & 5 \\
\hline apérat. . & 0,5 & 0,6 & 0,5 & 0,5 & 1,0 & 0,8 & 0 & 0 \\
\hline
\end{tabular}

Les groupes A, B, C et D du tableau indiquent les différentes conditions dans lesquelles la montée de la crème a eu lieu. Dans le groupe A, la proportion entre la hauteur et le diamètre de la couche de lait est dans le rapport 1 à 1 ; dans le groupe $\mathrm{B}, 2$ à 1 ; etc..., ce qui revient à dire que la montée de la crème a été étudiée dans des couches de lait très différentes comme hauteur. Tous les chiffres sont des moyennes d'un certain nombre d'essais ; mais, dans un but de comparaison, il ne faut prendre que les chiffres des colonnes verticales, car les chiffres des divers groupes ne proviennent pas du même lait, mais de prises d'échantillons à des heures différentes.

Le tableau montre que la montée de la crème n'est pas aussi forte dans le lait stassanisé que dans le lait brut; mais qu'elle est très supérieure à celle du lait pasteurisé à haute température. La raison de la plus faible montée de la crème peut certainement être attribuée au fait que, pendant le passage à travers la tuyauterie longue et étroite, a lieu une certaine homogénéisation qui réduit les globules de matière grasse et qui rend difficile leur ascension à travers le sérum du lait. 
Ceci est particulièrement net quand la hauteur de la couche de lait est grande par rapport au diamètre comme dans les groupes $\mathrm{C}$ et $\mathrm{D}$.

L'écrémage par écrémeuse n'a donné lieu à aucune difficulté. Les essais a vec une écrémeuse à main et une écrémeuse méca nique ont donné le même résultat, c'est-à-dire un écrémage qui, dans le lait ordinaire, laissait $0,07 \%$ de matière grasse et $0,08 \%$ dans le lait stassanisé.

Lait ordinaire

Echantillon 1

0,06
$2 \ldots \ldots \ldots \ldots \ldots \ldots, 0,07$

$3 \ldots \ldots \ldots \ldots \ldots, 0,08$

$4 \ldots \ldots \ldots \ldots \ldots \ldots, 0,07$

$5 \ldots \ldots \ldots \ldots \ldots \ldots, 0,08$

6

0,06

Lait stassanisé

\section{$0,07 \%$ de}

matière grasse

0,07

0,08

0,08

0,08

0,09

0,08

La différence des résultats dans l'écrémage n'est pas plus grande qu'une différence occasionnelle. Dans l'ensemble, l'écrémage est un peu inférieur à la normale, ce qui, toutefois, peut être dû au fait que les conditions d'écrémage pendant ces essais n'ont pas été tout à fait les mêmes que pendant la marche journalière normale.

LA CONSERVATION dU LAIT STASSANisE. - Cette question a été étudiées dans un très grand nombre de cas comparativement avec du lait brut et avec du lait ordinaire pasteurisé à haute température $\left(90^{\circ}\right.$ C. $=194^{\circ} \mathrm{F}$.). Le résultat de ces essais est que les divers échantillons de lait, après avoir été chauffés et refroidis à des températures variant entre $10^{\circ}$ et $15^{\circ} \mathrm{C}$. $\left(50^{\circ} 0\right.$ à $59^{\circ} 0 \mathrm{~F}$.) ont montré les propriétés de conservation relevées dans le tableau ci-dessous après repos en ballons stériles bouchés a vec de l'ouate à la température de $16^{\circ}$ à $20^{\circ}$ C. $\left(60^{\circ} 8\right.$ à $68^{\circ} 0$ F. $)$ :

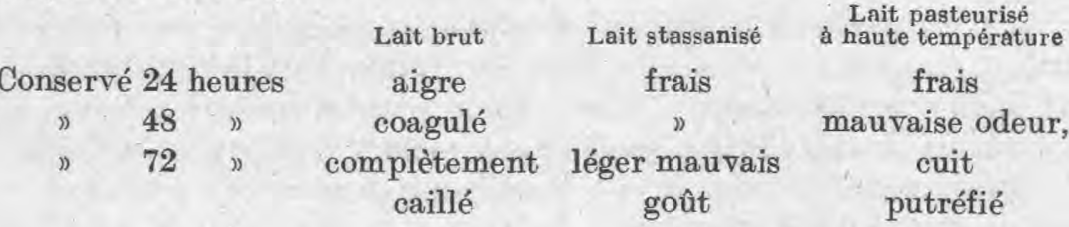

Pendant tous les essais, le lait stassanisé s'est conservé frais et sans goût désagréable pendant $\mathbf{4 8}$ heures̉, alors qu'aussi bien le lait brut que le lait pasteurisé à haute température devenaient respectivement aigres et se coagulaient, ou bien prenaient un mauvais goût très déplaisant. Après 72 heures, le lait stassanisé avait aussi un assez mauvais goût, quelque peu métallique, mais ses propriétés de conservation ont toujours été supérieures à celles des deux autres laits. 


\section{COMPOSITION CHIMIQUE DU LAIT STASSANISÉ}

Pendant les essais, divers éléments de la composition ont été étudiés à plusieurs reprises, et les résultats de ces examens sont indiqués dans le tableau ci-dessous qui indique la composition du lait à l'état brut et après chauffage :

\begin{tabular}{|c|c|c|c|c|c|c|c|c|}
\hline Echantillon & Lait & 1 & 2 & 3 & 4 & 5 & 6 & 7 \\
\hline \multirow{2}{*}{ Matière grasse \% } & Brut ......... & 4,17 & 3,87 & 3,71 & 3,21 & 3,58 & 3,60 & 3,45 \\
\hline & Stassanisé ... & 4,22 & 3,86 & 3,77 & 3,22 & 3,57 & 3,59 & 3,45 \\
\hline \multirow{2}{*}{ Azote total $\times$} & Brut ........ & - & 3,58 & - & - & 一 & 2,97 & 3,51 \\
\hline & Stassanisé ... & - & 3,54 & - & - & 一 & 3,04 & 3,41 \\
\hline \multirow{2}{*}{$\begin{array}{c}\text { Azote de la caséi- } \\
\text { ne dans le } \% \text { de } \\
\text { l'azote total }\end{array}$} & Brut ........ & - & 79,9 & - & - & 一 & 79,3 & 80,1 \\
\hline & Stassanisé ... & - & 81,1 & - & - & - & 80,8 & 81,2 \\
\hline Degré d'acidité & Brut ......... & 6,2 & 6,4 & 6,3 & 6,5 & 6,5 & 5,5 & 5,9 \\
\hline (Soxhlet-Henk.) & Stassanisé ... & 6,2 & 6,5 & 6,3 & 6,2 & 6,3 & 5,5 & 5,9 \\
\hline \multirow{2}{*}{$p \mathrm{H}$} & Brut ......... & 6,65 & 6,57 & 6,64 & 6,46 & 6,58 & - & 6,53 \\
\hline & Stassanisé ... & 6,67 & 6,60 & 6,62 & 6,62 & 6,58 & - & 6,58 \\
\hline 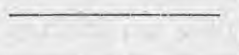 & & & & & & & & \\
\hline
\end{tabular}

Le tableau montre que le chauffage n'a modifié que très légèrement la composition du lait, mais on a trouvé une plus grande proportion d'azote de la caséine dans le lait stassanisé que dans le lait brut, ce qui doit certainement être attribué à la séparation de l'albumine et de la globuline, car des études spéciales de la proportion de ces eomposés azotés ont montré une diminution d'environ $8 \%$ dans le lait stassanisé.

A cet égard, on a aussi étudié l'influence de la stassanisation sur la faculté naturelle de coagulation du lait, aussi bien au laboratoire que dans la pratique courante de la fabrication du fromage.

Les essais de lahoratoire ont dönné les durées suivantes de coagulation :

Lait brut : 500 secondes,

Lait stassanisé : 568 secondes,

d'où il résulte que les chiffres proportionnels pour la faculté de coagu- 
lation pouvent être respectivement fixés à 9.600 et à 8.500 pour le lait brut et le lait stassanisé.

La différence ainsi trouvée au laboratoire pour les facultés de coagulation des laits bruts et chauffés n'a pas été retrouvée dans la pratique fromagère ou du moins elle a été trouvée si minime qu'elle n'a pas d'importance au point de vue pratique. Il faut toutefois ajouter que les essais pratiques de coagulation du lait stassanisé ont été peu nombreux en raison du peu de capacité de l'appareil qui faisait que le temps nécessaire au caillage d'une cuve de lait était si long qu'il était difficile de l'effectuer en même temps que le travail ordinaire de la laiterie. Pendant les essais de caillage, on n'a pas employé de chlorure de ealcium.

\section{RÉSISTANCE DES VITAMINES DU LAIT A LA STASSANISATION}

Comme la Laiterie d'Essais n'a pas à sa disposition les moyens de procéder à des recherches dans cette direction, elle a demandé au Professeur L.-S. Fredericta, Docteur en Médecine de l'Institut d'Hygiène de l'Université, de procéder aux recherches relatives à l'influence de la stassanisation sur les vitamines A, B et C. Le Professeur FredeRICIA accepta avec plaisir et prépara le programme de recherches suivant :

Pour chaque vitamine, deux séries d'essais sur animaux doivent être effectuées en même temps, une avec du lait non traité et l'autre avec du lait chauffé.

En conséquence, tous les jours (sauf le dimanche), la Laiterie d'Essais d'Hillerod enverra à l'Institut d'Hygiène de l'Université, 11, Ny Vestergade, à Copenhague, 2 litres (env. 0 gallon 44) de lait doux non traité et 2 litres (env. 0 gallon 44 ) du même lait doux après traitement dans l'appareil Stassano. Les échantillons de lait devront être accompagnés d'une note indiquant leur pourcentage en matière grasse (méthode Gerber).

$1^{0}$ Les essais, en ce qui concerne la vitamine A, seront effectués sur des rats. 2 séries d'essais sont à effectuer, chacune sur 15 animaux divisés en 5 groupes. Méthode suivie : de jeunes rats ayant un poids initial de 30-45 grammes seront nourris avec une ration ne contenant pas de vitamine $\mathrm{A}$, mais qui sera complète à tous autres points de vue. Cette ration de base consiste en : $20 \%$ caséine purifiée, $52 \%$ amidon de riz purifié, $15 \%$ graisse de porc purifiée, $5 \%$ d'un mélange de sels et $3 \%$ d'agar-agar. Chaque rat aura sa propre cage, et on notera la quantité de la ration de base mangée journellement par chaque animal. Quandla croissance de l'animal, a près 4-5 semaines, aura cessé pa.r suite de l'absence de vitamine A dans la ration, une quantité déterminée de lait sera ajoutée à la ration de base, un certain nombre d'animaux recevant du lait brut, les autres du lait stassanisé, et on notera la 
quantité de lait nécessaire pour que la eroissance des animaux reprenne à nouveau. De ces observations, on tirera des conclusions pour déterminer la contenance en vitamine A du lait. La période d'essai est estimée à 5 mois.

$2^{\circ}$ Las recherches relatives à la vitamine $\mathrm{B}$ seront effectuées sur des rats. La méthode sera la même que celle employée pour déterminer la vitamine A, mais la durée de ces recherches sera plus courte. Par contre, elles sont, pour la plupart, plus compliquées. C'est pourquoi 20 animaux seront utilisés pour chacune des 2 séries de recherches. La durée des recherches est estimée à 2 à 3 mois.

$3^{0}$ Los recherches relatives à la vitamine $\mathrm{C}$ seront effectuées avec des cobayes d'après la méthode décrite par le Professeur Axel HöYER, de Lund, dans The British Journal of Experimental Pathology 1926, Vol. VII, p. 356, qui est basée sur des recherches histologiques des modifications pathologiques des os des animaux. 10 cobayes seront employés pour chaque série de recherches, et la durée de ces recherches est estimée à 3 mois.

Ires recherches furent effectuées sur ces bases de mai 1928 à février 1929, et le Professeur FrIDERICIA en a donné les conclusions suivantes :

Résultats des essais relatifs à l'effet de la stassanisation sur la teneur en vitamines du lait doux :

10 La teneur en vitamine $\mathrm{A}$ du même lait a vant et après la stassanisation a été étudiée sur 6 groupes de rats, au total sur 26 animaux. Comme ration de base, était employé un mélange d'aliments qui ne contenait pas de vitamine A, mais qui, d'un autre côté, contenait tous les aliments nécessaires à de jeunes rats. Un des groupes était un groupe de contrôle qui a reçu la ration de base ne contenant pas de vitamine $\mathbf{A}$, tandis que l'autre était un groupe de contrôle nourri avec la ration de base, additionnée d'une ration journalière de $0 \mathrm{gr}$. 30 de beurre par animal pour produire une croissance normale des animaux. Chacun des 4 autres groupes fut divisé en 2 séries, dont l'une reçut, après que la croissance fut arrêtée, une addition de lait brut à la ration de base, l'autre une addition de lait stassánisé. Les 4 groupes reçurent journellement, à titre de supplément de vitamine $\mathrm{A}$, respectivement 0 ee 5 $-1 \mathrm{ce}-2 \mathrm{ce}-\mathrm{et} 3 \mathrm{ce}$ de lait.

La croissance des animaux de 4 séries qui avaient reçu du lait stassanisé a correspondu à celle des animaux qui avaient reçu du lait brut. 0 cc. 5 n'a rien produit ; 1 cc, a produit une petite croissance ; $2 \mathrm{cc}$. a produit une croissance presque normale; et $3 \mathrm{cc}$. une croissance tout à fait normale.

On peut en conclure que la teneur en vitamine A du lait a été de l'ordre de grandeur habituel, et n'a pas été modifiée d'une façon sensible par la stassanisation.

$2^{\circ}$ La teneur en vitamine B des mêmes échantillons de lait, a vant et 
après la stassanisation, a été étudiée sur 10 groupes de rats, au total sur 42 animaux. La méthode a été la même que celle décrite ci-dessus, pour la recherche de la vitamine A, avec cette exception que la ration de base employée ne contenait pas de vitamine B, mais, par contre, contenait tous les aliments nécessaires. Chacun des 8 groupes d'essai était divisé en 2 séries, recevant respectivement du lait brut et du lait stassanisé. Les 8 groupes expérimentaux furent chacun divisés en 2 séries recevant respectivement du lait brut et du lait stassanisé. Les divers groupes reçurent, après arrêt de la croissance, le supplément journalier de lait suivant pour chaque animal : $1 \mathrm{ce} .-2 \mathrm{cc} .-3 \mathrm{cc} .-$ 5 cc. -6 cc. -8 cc. -10 ce. et 12 cc.

Los animaux des groupes recevant 8 ce de lait par jour et moins n'augmentèrent pas. Las groupes avec addition de 10 ec et de $12 \mathrm{cc}$ grandirent normalement, et la croissance des séries recevant du lait stassanisé fut égale à celle des séries recevant du lait brut.

On peut en conclure quela teneur en vitamine $B$ du lait a été normale, mais à la limite inférieure extrême de la normale, et que la teneur en vitamine B n'a pas été modifiée par la stassanisation.

$3^{\circ}$ Vitamine C. 2 séries de recherches furent effectuées sur des cobayes, mais comme il fut difficile d'obtenir des animaux qu'ils boivent les quantités de lait nécessaires, on ne peut pas tirer de conclusion de ces séries d'essais.

\section{INFLUENCE DEYLA STASSANISATION SUR L'ETTAT BACTERIOLOGIQUE DU LAIT}

1. BACTERIES ORdinaIRES du LAIT. - Par analogie à ce qui avait été fait lors de la pasteurisation basse et de la pasteurisation lente du lait pour la fabrication du fromage (voir le $5^{\mathrm{e}}$ rapport), on a aussi étudié, à l'occasion de la stassanisation, dans les essais ci-contre, l'effet de ce traitement sur la teneur en bactéries. C'est pourquoi on a effectué une série d'ensemencements, sur plaques de gélatine en boîtes de Piétri, du même lait avant et après stassanisation.

Le mllieu alimentaire employé a été, soit le suivant indiqué par le Professeur ORLA-Jensen :

120 gr. gélatine

20 " peptone Witte

10 ) glucose

10 "lactose

1 ) sulfate de magnésium

2 ") sulfate de potassium

2,5 sel ordinaire

1000 " d'eau, soit de l'agar-sérum pur.

Les plaques furent généralement conservées pendant 7 jours et 
7 nuits dans un thermostat à $20^{\circ}-22^{\circ} \mathrm{C}\left(68^{\circ}-71^{\circ} 6 \mathrm{~F}\right.$.). Dans quelques cas, les ensemencements restèrent pendant 3 jours-à une température de $30^{\circ} \mathrm{C}$. $\left(86^{\circ} \mathrm{F}\right.$.), ce qui n'eut, du reste, aucune influence sur les résultats. La numération des plaques, a près les périodes ci-dessus mentionnées, a donné les résultats suivants :

Numération DES BaCtérIES PAR CC. DE LAIT BRUT ET STASSANISÉ

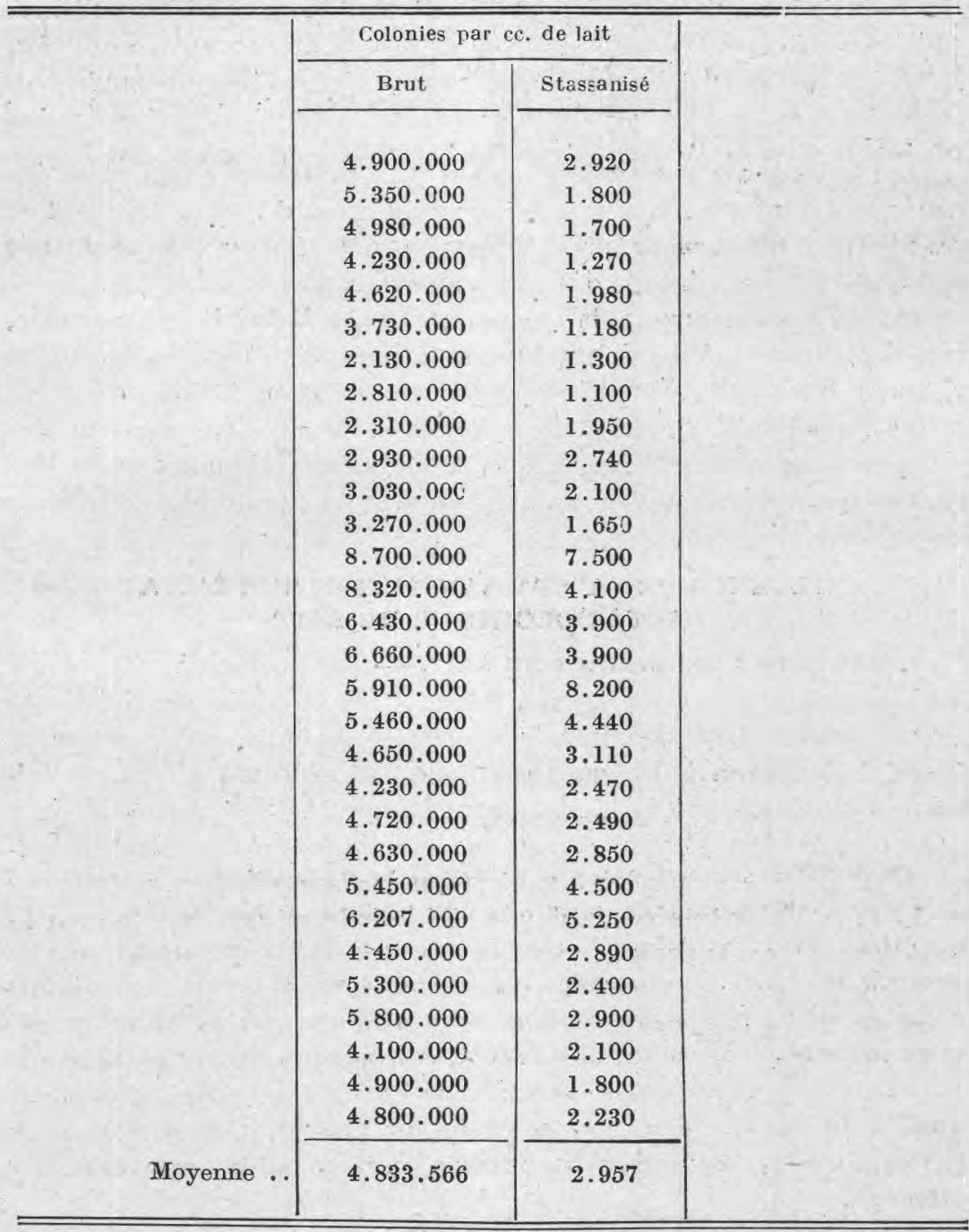

Effet de la pasteurisation : 99,94\%(1).

(1) Les effets de la pasteurisation dépendent naturellement beaucoup de la teneur initiale en bactéries du lait brut. 
L'effet de la pasteurisation a été en moyenne de $99,94 \%$, avec de petites variations.

Avant ces essais, l'appareil fut très soigneusement nettoyé et, après le nettoyage ordinaire, il fut entièrement passé à la va peur, et, le matin, lavé soigneusement à l'eau bouillante avant usage.

Si ces précautions ne sont pas prises, ou si on laisse se développer des incrustations dans les tuyaux, l'effet de la pasteurisation sera diminué, ce qui est prouvé par le tableau suivant dont les résultats proviennent d'échantillons de lait prélevés sur l'appareil a près quelques jours d'emploi sans que des mesures spéciales de nettoyage aient été prises, en dehors d'un nettoyage à l'eau bouillante le matin avant que l'appareil ne soit employé.

NUMÉration SUR PLAqUES DES BaOtérIES DANS DU LAIT BRUT ET STASSANISÉ

\begin{tabular}{cc}
\multicolumn{2}{c}{ Colonies par ce. de lait } \\
Brut & Stassanisé \\
9.670 .000 & 44.000 \\
9.430 .000 & 58.000 \\
8.700 .000 & 37.500 \\
8.320 .000 & 46.500 \\
7.600 .000 & 41.000 \\
8.100 .000 & 48.000 \\
4.420 .000 & 50.500 \\
3.970 .000 & 24.200 \\
3.230 .000 & 28.420 \\
3.110 .000 & 28.960 \\
2.870 .000 & 18.210 \\
3.340 .000 & 22.600 \\
\hline Foyenne: 5.980 .000 & \\
Ffet la pasteurisation : $99,38 \%$. &
\end{tabular}

On voit dans ce tableau que l'effet de la pasteurisation a été réduit à 99,38\%. Evidemment, le nombre des bactéries qui ont survécu au chauffage n'est pas particulièrement grand si on le compare au nombre primitif des bactéries contenues dans le lait brut. Du reste, leur vitalité a été en même temps très sérieusement affaiblie par le chauffage. On peut toutefois souligner qu'un nettoyage soigneux, ou un passage à la vapeur, de l'appareil est de très grande importance pour maintenir la qualité du lait traité et, comme on l'a dit plus haut, le nettoyage de l'appareil n'est pas tellement difficile qu'il ne puisse être très bien effectué.

Naturellement, ce nettoyage exige des soins et de la conscience de la part de la personne qui doit l'effectuer, mais il ne peut pas en être autrement. Si on veut jouir de l'avantage d'avoir le lait traité sans admission d'air - et ceci est certainement un très grand avantage - 
il est, naturellement, nécessaire d'employer des tuyaux fermés et de rendre ceux-ci aussi faciles à démonter que possible en vue de leur nettoyage. Ces prescriptions ont été suivies, aussi loin qu'on l'a pu, dans la construction du nouvel appareil à stassaniser.

- Les résultats ci-dessus mentionnés justifient l'opinion que le chauffage du lait entrepris par l'emploi de l'appareil à stassaniser fait du lait un aliment sain qui se conserve, car ce chauffage tue ou affaiblit fortement la flore bactérienne très importante et très étendue qu'on trouve toujours, dans les conditions ordinaires, dans le lait brut.

2. BACTÉRIES PATHOGÉNES DU LAIT. - Outre la flore bactérienne généralement présente dans le lait, dont la plus grande partie est normalement plutôt inoffensive, et qui limite son activité à une transformation appropriée du lait qui a lieu plus ou moins tôt, il y a aussi, comme on le sait, des bactéries qui peuvent produire des maladies quand elles sont amenées par le lait dans d'autres organismes animaux. De telles bactéries peuvent se trouver dans le lait en provenance d'animaux malades ou de personnes traitant le lait. Elles peuvent aussi occasionnellement provenir d'autres milieux avec lesquels le lait est en contact.

C'est pourquoi il est naturel que les consommateurs de lait exigent d'être tout à fait garantis contre la transmission par le lait qu'ils achètent de maladies infectieuses, par suite de la présence dans ce lait de bactéries pathogènes.

Le chauffage du lait est très utile pour l'obtention d'une telle garantie, et c'est pourquoi, en même temps qu'on étudiait l'influence de la stassanisation sur l'état bactériologique du lait, on á aussi procédé à des recherches dans le but de savoir comment elle agissait sur les bactéries du groupe Coli-Aerogenes :

Sans savoir si oui ou non le lait brut contenait des bactéries de cette nature, on a procédé à une série d'essais à ce sujet sur le lait stassanisé. On a versé des échantillons de ce lait dans des bouteilles stériles qu'on a laissé reposer pendant 24 heures à une tem pérature de $38^{\circ} \mathrm{C}$. (100 $4 \mathrm{~F}$.). Avec ces laits, on a ensuite procédé à des ensemencements sur plaques, soit sur glucose, soit sur peptone-agar, soit sur sérum-agar ; mais, dans aucun cas, on n'obtint de développement de colonies de coli ou de bactéries aérogènes.

On a alors préparé une culture vigoureuse de ces bactéries. 4 litres (env. 0,89 gallon) de cette culture furent ajoutés à 100 litres (env. 22,2 gallons) de lait ordinaire, et on en prit un échantillon pour procéder à des ensemencements. Le lait fut ensuite envoyé dans l'appareil et chauffé à la manière habituelle jusqu'à $75^{\circ} \mathrm{C}$. (1670 F.) pendant 15 16 secondes. De ce lait, fut aussi prélevé un échantillon pour ensemencements. 
Après une période de repos convenable, les deux ensemencements des 2 laits furent examinés : celui du lait brut présentait un développement important de bactéries coli, tandis que celui du lait stassanisé ne présentait pas trace de colonies appartenant audit groupe.

La stassanisation avait donc bien détruit toutes les bactéries du groupe Coli-Aerogenes.

Pendant que s'effectuaient à la Laiterie d'Essais les recherches sur le chauffage du lait par la méthode du $\mathrm{D}^{\mathrm{r}}$ Stassano, le Professeur P. BANG, de l'Esole Supérieure d'Agriculture et de Médecine Vétérinaire, exécutait, lui aussi, très aimablement, des recherches sur la valeur de ce chauffage en ce qui concerne la destruction des bactéries de l'avortement épizootique (Bacillus abortus BANG) et les bacilles de la tuberculose. Le Professeur BANG a présenté le rapport suivant qui décrit l'exécution de ses recherches et les résultats obtenus :

\section{a) Recherches sur la bactérie de l'avortement épizootique}

Le 31 mars 1928, la Laiterie d'Essais a mélangé 50 litres (11 gal. 1) de lait en provenance d'un troupeau dont on savait que plusieurs vaches produisaient du lait infecté par la bactérie de l'avortement épizootique avec 50 litres (11 gallons 1) de lait ordinaire. Sur ce mélange fut prélevé un échantillon de 2 litres (env. 0,44 gallon); le reste fut passé dans l'a ppareil de Stassano et chauffé à $75^{\circ} \mathrm{C}$. ( $167^{\circ} \mathrm{F}$.) pendant 16 secondes.

Sur le lait ainsi chauffé, fut prélevé un échantillon de 2 litres $(0,44$ gallon) qui fut versé en bouteilles stériles.

Avec les laits des échantillons ei-dessus furent inoculés le même jour des cobayes comme suit :

8 a vec de la crème et de la boue d'écrémeuse du lait brut infecté avant stassanisation, et

5 avec le même lait après stassanisation.

Après 3 mois, les résultats suivants ont été constatés :

Des 8 cobayes inoculés avec le lait infecté, 2 sont morts des suites de l'inoculation. 1 mourut plus tard pour une cause inconnue, et une mourut le 24 mai, probablement d'infection par le Bacille de BANG, de l'a vortement infectieux ; dans tous les cas, des B. abortus furent trouvés dans la rate. Quand on tua les 4 derniers animaux de contrôle, on les trouva tous fortement atteints d'avortement infectieux.

Das 5 cobayes inoculés avec le lait stassanisé, un mourut pour une cause accidentelle; les 4 autres furent tués a près 2 mois, et trouvés tous sains.

Résultat : tous les animaux de contrôle furent nettement atteints ; aucun de ceux inoculés avec le lait stassanisé ne fut touché.

\section{b) Recherches sur le bacille de ia tuberculose}

Des laits de 4 vaches différentes ayant de la tuberculose du pis furent utilisés. 
ler essai ; le 16 mai 1928, 2 litres (env. 0,44 gallon) de lait d'une glande galactifère tuberculeuse d'une vache ayant de la tuberculose du pis furent mélangés avec 40 litres. (env. 8,8 gallons) de la Laiterie d'Essais, et un échantillon de 2 litres (env. 0,44 gallon) fut prélevé sur le mélange. Le mélange de lait restant fut stassanisé à $75^{\circ} \mathrm{C}$. (167\% F.) pendant $15-16$ secondes, a près quoi un échantillon de 2 litres ( 0,44 gallon) fut de nouveau prélevé.

Le même jour, 4 cobayes furent inoculés avec de la boue d'écrémeuse traitée au formol provenant de chacun des 2 échantillons de lait. La tuberculose ne se développa pas sur les animaux inoculés avec le lait stassanisé ; ils furent tués le 29 octobre, tandis que tous les animaux de contrôle inoculés avec du lait infecté furent atteints de tuberculose, mais 2 vécurent jusqu'au 29 octobre 1928, date à laquelle ils furent tués et trouvés très tuberculeux.

$2^{\text {e }}$ essai : le 29 novembre 1928 , la Laiterie d'Essais mélangea 5 litres (env. 1,11 gallon) du lait d'une vache ayant de la tuberculose du pis a vec 40 litres (env. 8,8 gallons) de lait de la Laiterie d'Essais. 2 échantillons furent prélevés sur le mélange, et le reste passa à traversl'appareil à stassaniser, a près quoi 2 autres litres (env. 0,44 gallon) du lait traité furent prélevés a près qu'il eût été chauffé à $75^{\circ} 1 / 4 \mathrm{C}$. (env. 16701 F.).

Le même jour, 3 cobayes furent inoculés sous la peau arec $2 \mathrm{ec}$. du lait chauffé comme indiqué ci-dessus. Les cobayes furent tués le 23 mai 1929. Aucun ne présentait de trace de tuberculose, et ils étaient aussi parfaitement sains à tous autres égards.

En même temps, 3 cobayes furent aussi inoculés avec le lait brut infecté mélangé du 29 novembre 1928 , et ces animaux de contrôle furent respectivement inoculés a vec $2 \mathrm{cc}$. et $1 \mathrm{ec}$. de lait brut. Tous ces animaux moururent de tuberculose dans les 48 jours suivant l'inoculation,

$3^{\text {e }}$ essai : le 28 décembre 1928,4 litres $1 / 2$ de la sécrétion très modifiée des glandes galactifères d'une vache ayant de la tubereulose du pis furent mélangés, à la Laiterie d'Essais, avec 40 litres de lait (env. 8,88 gallons), et les échantillons habituels furent prélevés sur le lait avant et après la stassanisation.

3 cobayes furent inoeulés avec le mélange de lait après chauffage, chacun avec $2 \mathrm{ec}$. du lait chauffé. Le 29 janvier 1929, on examina les cobayes. Tous étaient sains sans trace de tuberculose. 2 des animaux moururent ultérieurement fortuitement le 13 a vril 1929 et le 6 mai 1929 ; le $3^{\mathrm{e}}$ animal fut tué le 30 mai 1929. Aucun de ces trois eobayes n'avait de trace de tuberculose.

Le même jour que plus haut ( 28 décembre 1928 ) 3 cobayes de contrôle furent inoculés avec le mélange de lait avant chauffage, chaque cobaye étant inoculé a vec $1 \mathrm{cc}$. de lait brut sous la peau (les animaux de contrôle ne furent donc inoculés qu'a vec moitié en lait brut de la dose 
avec laquelle les animaux d'expérience avaient été inoculés) en lait chauffé. Les animaux de contrôle moururent tous de tuberculose respectivement les 25 janvier, 12 mars et 13 a vril 1929.

11 faut faire remarquer ici qu'en réalité on a inoculé environ le double de cobayes que ceux dont il est question ici, car on a inoculé des cobayes a vec de la boue d'écrémeuse formolée provenant des échantillons de lait prélevés, afin d'être sûr que les cobayes ne mourraient pas d'une infection provenant de l'inoculation. Les résultats de ces inoculations ont été identiques à ceux indiqués plus haut.

$4^{\mathrm{e}}$ essai : le 5 janvier 1929, on a mélangé à la Laiterie d'Essais 4 litres (environ 0,88 gallon) de sécrétion laiteuse d'une vache ayant de la tuberculose du pis avee 40 litres (env, 8,88 gallons) de lait ordinaire.

Dəs éshantillons furent prélevés sur ce mélange de lait avant et après le chauffage.

Le 5 janvier 1929, 3 cobayes furent chacun inoculés avec $2 c c$. $d u$ mélange de lait après chauffage de celui-ci à $75^{\circ} \mathrm{C}$. (1670 F.). Un cobaye mourut le 12 avril 1929 d'une cause fortuite et sans trace de tuberculose. Las 2 autres furent tués le $1^{\text {er }}$ juillet 1929 ; tous les 2 étaient gras et sans signe de tuberculose.

3 autres cobayes furent inoculés a vec la boue d'écrémeuse de $100 \mathrm{cc}$. de lait stassanisé. Cette boue avait été traitée avec une solution à $10 \%$ de formol. Un de ces animaux mourut d'une maladie fortuite le 23 mai 1929 : il n'avait pas de tuberculose. 2 furent tués le 1 er juillet 1929. Ils étaient tous deux gras et sans signe de tuberculose.

Avant que le chauftage n'ait été effectué, 3 cobayes furent inoculés avec chacun 1 ce. de lait brut. Tous moururent de tubereulose du 13 mars au 2 avril 1929. 3 autres cobayes furent inoculés avec la boue d'écrémeuse de $100 \mathrm{cc}$. de lait brut ; la boue d'écrémeuse a vait été traitée pendant $1 / 2$ heure avec une solution à $10 \%$ de formol.

Ces derniers cobayes moururent de tubereulose du 21 mai au 18 juin 1929.

Des expériences effectuées par le Professeur BANG, on peut conclure que le chauffage du lait qui a lieu dans l'appareil à stassaniser a été susceptible de détruire effectivement aussi bien les bacilles de l'a vortement que les bactéries de la tuberculose, et, en conséquence, que le lait ainsi traité remplit toutes les conditions convenables en ce qui concerne l'hygiène.

\section{CONTROLE DU CHAUFFAGE}

Quand le lait est chauffé à $80^{\circ} \mathrm{C}$. ( $\left(176^{\circ} \mathrm{F}\right.$.) ou plus, une modification de sa composition chimique a lieu, comme il a été indiqué ci-dessus, car une des enzymes (la peroxydase) du lait est détruite à une température de 79 à $80^{\circ} \mathrm{C} .\left(174^{\circ} 2-176^{\circ} \mathrm{F}\right.$.). Ce phénomène a été, on le sait, étudié en son temps par le Professeur SToRch qui a basé sur lui 
le contrôle de la pasteurisation du lait, contrôle qui est reconnu et adopté partout comme une preuve du chauffage du lait à $80^{\circ} \mathrm{C}$. et plus.

Dans le cas du chauffage du lait effectué à des températures plus basses, soit dans le cas de la pasteurisation lente à $63^{\circ} \mathrm{C}$. (14504 F.) pendant 30 minutes, soit dans celui des essais ci-dessus de la méthode Stassano à $75^{\circ}$ C. $\left(167^{\circ} \mathrm{F}\right.$.) pendant 15 secondes, la réaction du Professeur Stonor ne peut pas être utilisée pour le contrôle de la température, car la transformation du lait n'a pas été suffisante pour détruire la peroxydase, et, par conséquent, le lait chauffé à ces températures se colorera en bleu lors de la réaction de STORCH, comme le lait brut.

On a essayé de modifier la réaction de STORCH en tenant compte de ce qu'il avait dit en son temps en ce qui concerne l'utilisation de cette réaction pour les laits ayant une acidité supérieure à la normale.

Un certain nombre d'autres réactions, comme celle de SCHARDINGER, etc., ont aussi été essayées, a près modifications, mais les résultats de ees essais ont été tels qu'on ne peut pas les indiquer comme constituant actuellement des méthodes de contrôle sur lesquelles on puisse compter avec certitude. Il est probable que, dans l'ensemble, il sera difficile de trouver une réaction suffisamment rapide et pratique du contrôle d'un chauffage qui, pratiquement, ne modifie le lait brut qu'au point de vue bactériologique.

La faculté un peu amoindrie de montée de la crème que le lait stassanisé a montré lorsqu'on le compare à du lait brut ne semble pas, d'après les essais effectués ici, être suffisamment régulière pour permettre son utilisation comme contrôle du chauffage du lait à exactement $75^{\circ} \mathrm{C}$. ( $167^{\circ} \mathrm{F}$.), car elle dépend en particulier de la plus ou moins forte homogénéisation subie par le lait pendant son passage à travers l'appareil.

Par l'utilisation d'un thermomètre enregistreur sur l'appareil, on peut toutefois contrôler que le lait a été chauffé à la température exacte, de même qu'un examen bactériologique donnera aussi un renseignement à ce sujet, mais ce dernier procédé est, bien entendu, plutôt diffieile à utiliser dans la pratique ordinaire.

\section{RÉSUME}

Les résultats des essais mentionnés dans l'exposé ci-dessus sur le traitement par chauffage du lait selon le procédé du docteur Stassano au moyen de l'appareil montré dans la figure 1, livré et monté par les usines de Silkeborg (Silkeborg Maskinfabrik), sont les suivants :

Comme le traitement se fait dans un système de tuyaux fermés, la teneur naturelle en acide carbonique, en sels calcaires et en phos phates ne varie pas pendant le chauffage, de même qu'aucune vaporisation du liquide ne se produit, et le traitement se fait par conséquent sans perte.

Le traitement par chauffage proprement dit a été fait à une tempé- 
rature de $75^{\circ} \mathrm{C}$. et a duré de 15 à 16 secondes. Le temps total pour le passage à travers les trois parties de l'appareil a été de deux minutes et demie environ. Le traitement n'a altéré ni l'odeur, ni le goût natugrels du lait.

La montée de la crème du lait "stassanisé » a été un peu inférieure à celle du même lait avant le traitement, bien qu'elle ait été beaucoup supérieure à celle du lait pasteurisé à haute température.

L'influence du traitement sur la faculté du lait de se coaguler par la présure a été si minime qu'elle n'a pratiquement pas porté préjudice au caillage.

L'écrémage parfait au moyen d'une écrémeuse à main ou mécanique se fait aussi aisément avec le lait stassanisé qu'avec le lait cru.

Gardé dans des conditions identiques, le lait stassanisé se conserve plus longtemps que le lait cru et le lait pasteurisé a haute température.

La composition chimique du lait a été très peu influencée par le traitement ; environ $8 \%$ seulement des protéines dissoutes sont transformées.

La teneur du lait en vitamines A et B n'a pas été influencée par le traitement.

Le traitement a détruit de 99,4 à $99,9 \%$ de la flore bactérienne ordinaire du lait.

Le traitement a également tué tous les colibacilles, la bactérie de l'avortement épizootique et le bacille de la tuberculose.

La consommation en vapeur, en force et en eau par ce procédé a été inférieure à celle des procédés de la pasteurisation ordinaires.

I'appareil Stassano demande un nettoyage soigneux, mais ce dernier n'est point trop difficile ; il peut être effectué dans la pratique ordinaire, et la construction nouvelle de l'a ppareil montrée dans la figure 2 offrira probablement des avantages à cet égard.

(Traduction de M. C. WoLf.)

\section{LA MÉTHODE SYNTHÉTIQUE DANS L'ÉTUDE DU LAIT. ILE LAIT AU:POINT DE VUE COLLOIDAL. RECHERCHES SUR LE MÉCANISME IDE L'ACTION DE LA PRÉSURE}

par Ch. PORCHER,

Docteur ès sciences physiques

(Suite)

Notre première hypothèse sur le rôle des phosphates insolubles de calcium dans l'emprésurage. - Comme nous le

(257) Ch. Porcher. - Les divers complexes caséinate de chaux + phosphate de chaux et leur façon de se comporter vis-à-vis de la présure. C. R. Ac. Sc. 1925, 180, 1534. 